\title{
Hilft Vitamin C wirklich gegen Schnupfen?
}

\section{Viele Menschen schwören auf Vita- min C zur Prophylaxe und Therapie des Schnupfens.}

- Israelische Forscher randomisierten 39 Jugendliche, die regelmäßig für Schwimmwettkämpfe trainierten. Sie erhielten drei Monate lang täglich entweder 1 g Vitamin C oder Placebo. Die Häufigkeit der in diesem Zeitraum auftretenden Erkältungskrankheiten wurde durch diese Prophylaxe nicht reduziert. Auch die Länge des Schnupfens war im Durchschnitt nicht signifikant kürzer.

Allerdings ergab sich hier ein nicht signifikanter positiver Trend, der für die Vitamin-C-Gabe sprach. Weitere Analysen deuteten ebenfalls eine Wirksamkeit der Ascorbinsäure an. Bei den männlichen Studienteilnehmern waren die schnupfenverkürzenden Effekte signifikant. Die Autoren meinen, dass diese Ergebnisse erfolgversprechend sind und weiterer Forschung bedürfen.

\section{Kommentar}

Inzwischen gibt es zahlreiche Studien zum

Thema Schnupfenprophylaxe mit Vitamin C. Einige von ihnen scheinen das zu bestätigen, was die hier vorliegende, leider sehr kleine Studie andeutet: Die Erkältungsdauer kann durch regelmäßige Einnahme von hoch dosiertem Vitamin C verkürzt werden. Der Effekt ist jedoch nur marginal. Daher sind Studien mit niedrigen Fallzahlen auch wenig geeignet, uns hier erheblich weiterzuhelfen. Fazit: Vitamin C verhütet einen Schnupfen wohl nicht, kann ihn aber (um einige Stunden) abkürzen.

- N. W. Constantini et al.

The effect of vitamin $C$ on upper respiratory infections in adolescent swimmers: a randomized trial. Eur. J. Pediatr. 170 (2011) 59-63 